\title{
THE
}

$10-1-1984$

\section{Wetting and Growth Behaviors in Adsorbed Systems with Long- Range Forces}

\author{
M. P. Nightingale \\ University of Rhode Island, nightingale@uri.edu \\ W. F. Saam \\ M. Schick
}

Follow this and additional works at: https://digitalcommons.uri.edu/phys_facpubs

Terms of Use

All rights reserved under copyright.

\section{Citation/Publisher Attribution}

Nightingale, M. P., Saam, W. F., \& Schick, M.. 1984). Wetting and growth behaviors in adsorbed systems with long-range forces. Physical Review B, 30, 3830. doi: 10.1103/PhysRevB.30.3830

Available at: http://dx.doi.org/10.1103/PhysRevB.30.3830

This Article is brought to you for free and open access by the Physics at DigitalCommons@URI. It has been accepted for inclusion in Physics Faculty Publications by an authorized administrator of DigitalCommons@URI. For more information, please contact digitalcommons-group@uri.edu. 


\title{
Wetting and growth behaviors in adsorbed systems with long-range forces
}

\author{
M. P. Nightingale \\ Department of Physics, University of Washington, Seattle, Washington 98195 \\ and Department of Physics, University of Rhode Island, Kingston, Rhode Island 02881* \\ W. F. Saam \\ Department of Physics, Ohio State University, Columbus, Ohio 43210 \\ M. Schick \\ Department of Physics, University of Washington, Seattle, Washington 98195 \\ (Received 13 February 1984)
}

\begin{abstract}
The growth and possible wetting behaviors of an adsorbed film are studied employing a solid-onsolid model in the presence of a hard wall and external potential $V(h)$ which is of long range. The model is analyzed with the use of position-space renormalization-group methods within the Migdal approximation. The existence of wetting transitions and their nature depends on the asymptotic behavior of $V(h)$ at large distances. We find that critical wetting cannot take place in this model. From what is known of $V(h)$, we conclude that wetting can be observed only along the gas-liquid phase boundary; however, first-order transitions between thin and thick films, which may be experimentally difficult to distinguish from wetting, can be observed along any phase boundary. The nature of the global phase diagram depends on the form of $V(h)$ and several general behaviors are presented. In particular, in the layering subregime we find that the limit of layering critical points is indeed the bulk roughening temperature as had been suggested by de Oliveira and Griffiths. The scaling of these layering critical points is given explicitly.
\end{abstract}

\section{INTRODUCTION}

The growth of adsorbed films and the related wetting transition have been the subject of considerable theoretical and experimental attention recently..$^{1-3}$ In a typical experimental situation, a cell at temperature $T$. containing an adsorbate (substrate) is filled with a known amount of gas, some of which adsorbs on the substrate, forming a film of thickness $l$. As the amount of gas, and thereby its pressure, is increased, the thickness of the film is monitored and various transitions within the film are detected. Finally, as gas-solid or gas-liquid coexistence is encountered, the thickness of the film either approaches a finite limit, in which case the substrate is said to be "not wet" or "partially wet," or it increases without limit and the substrate is said to be "wet." The wetting transition, if it occurs, is the change from one behavior at coexistence to the other.

We consider the following mechanism which can lead to wetting. Suppose that the substrate is not wet at low temperatures for energy reasons, i.e., the net energy gained in putting a macroscopically thick film on the substrate which entails creating a liquid (solid)-substrate interface (energy gain) and a liquid (solid)-gas interface (energy cost) is less than that gained by putting on either no film, which entails only a gas-substrate interface, or a thin film. As the temperature is increased, the creation of an increasingly thicker film is entropically favored as such a film allows the existence of an increasing number of surface fluctuations. If the gain in entropy with thickness more than compensates the cost in energy, the thickness will grow without limit and wetting results.

The nature of the wetting transition depends crucially on whether the forces are short range (exponential decay) or long range (algebraic decay). In the former case, renormalization-group ( $R G)$ calculations have shown that the transition can be continuous or first order. ${ }^{2,4,5}$ If the wetting is continuous, denoted "critical wetting," the singularity in the surface free energy is nonuniversal., ${ }^{4,5}$ The upper critical (bulk) dimension is three. ${ }^{6}$ Some results of mean-field theory (MFT) are correct (e.g., that the transition can be either continuous or first order $^{7-10}$ ), others are not (e.g., that the singularities are classical).

In experimental systems, the forces are long range. In this case, as we reported earlier ${ }^{2}$ (see also Sec. III B 3), RG methods indicate that the above mechanism can only produce a first-order transition. The upper critical dimension is unknown for this case, although it has been speculated to be two for van der Waals forces. ${ }^{11}$

The nature of the global phase diagram has been explored by several authors. Three different models have been employed and have been examined via various methods. The models are as follows:

(i) Continuum models which have been examined solely by MFT. ${ }^{7,10}$

(ii) Lattice-gas models which have been studied using MFT (Refs. 9 and 12) and numerical simulation. ${ }^{13}$ Such models most often contain two phases, but two variants with three possible phases to simulate liquid, gas, and solid have been considered. ${ }^{14,15}$

(iii) Solid-on-solid (SOS) models which have been stud- 
ied by $\mathrm{RG}^{2,16}$ modified MFT, ${ }^{17}$ and finite-size scaling methods. ${ }^{18}$

The only global studies which have included realistic long-range adatom-adatom and adatom-substrate forces are the continuum model calculations employing MFT. ${ }^{7,10}$ By the nature of the model, these calculations do not address the interesting question of the effect of the roughening transition on the phase diagram. By the nature of the method, they cannot address the effect of the thermal fluctuations.

We have chosen to employ an SOS model specifically because the phenomenon of roughening can be included. ${ }^{19}$ Further, we can investigate the question of whether the limit of the layering critical points is the roughening temperature. ${ }^{12}$ We utilize real-space RG methods which entail an analysis in a very large parameter space, large enough to encompass the varied phenomena expected. Nonetheless, we show that the fixed-point structure and RG flows are quite simple. The model, the interactions, and the RG methods we have applied are discussed in Sec. II. The nature of the global phase diagram depends on the form of the potential between interface and substrate $V(h)$. Results for several different forms are presented in Sec. III according to the behavior encountered: $A$, wet at zero temperature; $B$, not wet at zero temperature. In the latter case, layering, prewetting, and critical wetting subregimes are discussed. The question of whether a wetting transition can occur depends only on the asymptotic behavior of $V(h)$. In Sec. IV we summarize what is known about $V(h)$ for adsorbed systems which, in conjunction with our results of Sec. III, enables us to discuss the possible wetting behaviors in such films.

\section{MODEL}

The SOS model is an obvious one to employ whenever an interface exists. Since the interface to be described is two dimensional, so is the lattice. On each of its sites exists an integer variable $h_{i} \geq 0$ which gives the height of the interface above the substrate at the site $i$. For technical reasons, we take the lattice to be triangular. To facilitate computation, we consider nearest-neighbor interactions only so that the Hamiltonian is

$$
\begin{aligned}
H-\mu M= & \sum_{\langle i j\rangle} W\left(\left|h_{i}-h_{j}\right|\right) \\
& +\sum_{i} V\left(h_{i}\right)-\left(\mu-\mu_{0}\right) h_{i},
\end{aligned}
$$

where $M \equiv \sum_{i} h_{i}$, and $\mu_{0}$ is the value of the chemical potential at coexistence. $W(h)$ is a monotonically increasing function of $h$ with $W(0)=0$. The first term, in which the sum is over all distinct nearest-neighbor pairs, represents the effect of the surface tension and is minimized when all $h_{i}$ are the same. The second term represents the interaction between the substrate and an interface a distance $h_{i}$ above it at site $i$.

As is well known, the SOS model is a special case of the lattice-gas model in which "islands" and "overhangs" are forbidden. Thus the $V\left(h_{i}\right)$ is easily related to the interac- tions of a lattice gas. Let us adopt the notation of Ref. 1 that $v_{s}$ is the interaction of an adatom with a single layer of adatoms a distance $s$ from it, and $w_{s}$ is the interaction of an adatom with a layer of substrate atoms a distance $s$ from it. Then the interaction of a single adatom a distance $m$ above a half-space of adatoms or of substrate atoms is

$$
t_{m}=\sum_{s=m}^{\infty} v_{s},
$$

or

$$
u_{m}=\sum_{s=m}^{\infty} w_{s}
$$

respectively. The energy of a uniform film of thicknesss $n$ is then easily put into the form of Eq. (2.1) with

$$
V(n)=\sum_{m=1}^{n}\left(u_{m}-t_{m}\right)
$$

so that $V(n)$ is the difference between the interaction of the adatoms in the film with the real substrate and a hypothetical substrate composed of adatoms. As all interactions are attractive ( $u$ and $t$ negative), it is seen that if adatom-substrate interactions are greater in magnitude than adatom-adatom interactions, $V(n)$ becomes more negative with increasing $n$. Thus the interface is repelled by the substrate. Conversely the interface is attracted if adatom-adatom interactions are greater in magnitude.

If coexistence is approached from the high-density side, then it is possible that a film of low-density gas of thickness $n$ is encountered. As the temperature is raised, the system can undergo a drying ${ }^{1}$ transition at which the thickness of the gas film diverges. The Hamiltonian of such a system can also be put into the form of Eq. (2.1) with

$$
V(n)=-\sum_{m=1}^{n} u_{m}+\text { const } .
$$

Thus the SOS model can be used for either circumstance provided that the interface potential is changed accordingly.

Although $V\left(h_{i}\right)$ in Eq. (2.1) can be related to interactions in a lattice gas, it need not be. That is, $V\left(h_{i}\right)$ can equally well describe interactions which cannot arise in a rigid lattice gas such as those induced by strain in a solid film. ${ }^{20-22}$

We note here that there is a lack of consistency in treating $V\left(h_{i}\right)$ as a long-range interaction while restricting the first term in the Hamiltonian to nearest-neighbor interactions. This restriction, which is necessary in order to make the RG calculation tractable, is not a serious one in terms of the physics of the problem, which only requires a surface tension to resist the deformation of the surface. The contribution of the long-range forces to the surface tension is not important and is irrelevant in the RG sense. ${ }^{23}$ However, the contribution of long-range forces to the interaction between interface and substrate is relevant and is included in our Hamiltonian. Other aspects of the SOS model which must be recognized is that it has no critical point at which the interface disappears and de- 
scribes two phases only. The former is a defect when the coexistence is between gas and liquid and the latter is a defect when the coexistence is between gas and solid.

We apply to the model of Eq. (2.1) simple positionspace RG methods within a Migdal approximation. ${ }^{24}$ To do this, we rewrite the Hamiltonian as a sum of terms associated with each bond between nearest-neighbor pairs

$$
H=\sum_{\langle i j\rangle} H\left(h_{i}, h_{j}\right)
$$

with

$$
\begin{aligned}
H\left(h_{i}, h_{j}\right)= & W\left(\left|h_{i}-h_{j}\right|\right) \\
& +\left[V\left(h_{i}\right)+V\left(h_{j}\right)-\Delta \mu\left(h_{i}+h_{j}\right)\right] / 6
\end{aligned}
$$

where $\Delta \mu \equiv \mu-\mu_{0}$. Note that

$$
H\left(h_{i}, h_{j}\right)=H\left(h_{j}, h_{i}\right) \text {. }
$$

We denote the Boltzmann weight ${ }^{25}$

$$
g_{0} x(h, k)=\exp [-H(h, k) / T],
$$

with $g_{0}$ to be defined below. Equation (2.4) implies the symmetry

$$
x(h, k)=x(k, h) .
$$

We employ a scale factor $b=2$ and shift bonds such that every other site can be decimated. This yields the simple recursion relation

$$
g x^{\prime}(h, k)=\sum_{m} x^{2}(h, m) x^{2}(m, k) .
$$

Note that the symmetry of Eq. (2.6) is preserved. The constant $g$ is determined by the normalization which we chose as follows. Let $s$ be the value of $h$ for which $x(h, h)$ is a maximum. [It is easy to see that this is the largest value of $x(h, k)$ in general.] We initially set this maximum weight to unity

$$
x(s, s)=1 .
$$

which defines $g_{0}$, and subsequently set the largest renormalized Boltzmann weight to unity. This largest weight can occur for a different value of the height, $s^{\prime}$ :

$$
x^{\prime}\left(s^{\prime}, s^{\prime}\right)=1 \text {, }
$$

This condition determines $g$ from Eq. (2.7) as

$$
g=\sum_{m} x^{4}\left(s^{\prime}, m\right)
$$

$$
\begin{aligned}
& A=\frac{\rho_{A} \sigma_{\mathrm{AA}}^{3}}{3}\left[\epsilon_{\mathrm{AA}} \rho_{A} \sigma_{\mathrm{AA}}^{3}-\epsilon_{\mathrm{AS}}\left(\rho_{S} \sigma_{\mathrm{AS}}\right)^{3}\left[\frac{\sigma_{A S}}{\sigma_{\mathrm{AA}}}\right]^{3}\right], \\
& B=-\rho_{A} \sigma_{\mathrm{AA}}^{3} \frac{29}{90}\left\{\dot{\epsilon}_{\mathrm{AA}} \rho_{A} \sigma_{\mathrm{AA}}^{3}-\frac{\epsilon_{\mathrm{AS}}\left(\rho_{S} \sigma_{\mathrm{AS}}\right)^{3}}{29}\left[\frac{\sigma_{\mathrm{AS}}}{\sigma_{\mathrm{AA}}}\right]^{3}\left[30-\left(\frac{\sigma_{\mathrm{AS}}}{\sigma_{\mathrm{AA}}}\right]^{6}\right]\right\},
\end{aligned}
$$

so that

$$
x^{\prime}(h, k)=\sum_{m} x^{2}(h, m) x^{2}(m, k) / \sum_{m} x^{4}\left(s^{\prime}, m\right)
$$

The Gibbs potential per site, $\Omega$, can be obtained from the constant piece $g$ in the usual way in the form

$$
\Omega(x) / T=3 \sum_{n=1}^{\infty} 4^{-n} \ln \left(g^{(n)}\right)+3 \ln g_{0},
$$

where the maximum Boltzmann weight on the $n$th iteration is $x^{(n)}\left(s^{(n)}, s^{(n)}\right)$. In the computations we approximated this expression by

$$
\begin{aligned}
\Omega(x) / T= & 3 \sum_{n=1}^{m} 4^{-n} \ln \left(g^{(n)}\right)+\ln \left(g^{(m)}\right) / 4^{m} \\
& +3 \ln g_{0} .
\end{aligned}
$$

This amounts to summing, rather than truncating, the series for $n>m$ under the assumption that $g^{(n)}$ is independent of $n$ for these $n$. Once the Gibbs potential is determined, the film thickness $l$ follows from $l=\partial \Omega / \partial \mu$.

As the column heights can, in principle, take on all nonnegative integer values, the matrix $x(h, k)$ is, again in principle, of infinite order. In practice we restrict the height variable $h$ to the range $0 \leq h<h_{\max }$ which produces a finite Hamiltonian space, invariant under renormalization, of $h_{\max }\left(h_{\max }+1\right) / 2$ nearest-neighbor couplings. The initial values of these couplings depend on the initial Hamiltonian Eq. (2.3). For the first term, we have chosen the form $W\left(\left|h_{i}-h_{j}\right|\right)=\left|h_{i}-h_{j}\right|$, which sets the energy of a step. For the potential $V(h)$ we have chosen

$$
V(h)= \begin{cases}-A / h^{2}-B, & h>0, \\ 0, & h=0 .\end{cases}
$$

The $h^{-2}$ behavior arises from the van der Waals forces in the problem. Note that with this choice of the zero of potential energy, $-\mathrm{B}$ is given by the infinite sum of the potential differences which appear in (2.2), where $A$ is related only to the long-range form of the difference. Consequently, $A$ and $B$ can be of different sign if the relative amplitude of the potentials at short distances is sufficiently different from that at large distances. The constants $A$ and $B$ can be related in some cases to the strength and range parameters of the adsorbate-substrate interaction $\epsilon_{\mathrm{AS}}, \sigma_{\mathrm{AS}}$, and adsorbate-adsorbate interaction $\epsilon_{\mathrm{AA}}, \sigma_{\mathrm{AA}}$. For example, if we assume structureless adatoms and the Lennard-Jones 6-12 form for these interactions, then where $\rho_{A}$ and $\rho_{S}$ are the densities of the adsorbed film and of the substrate. We stress that the above are merely useful for interpreting some circumstances under which $A$ and $B$ might be positive or negative. There are others in which these parameters are not given by the above. For example, consider those situations in which the substrate favors an adatom configuration in the first layer which differs substantially, in symmetry perhaps, from the con- 
figuration in bulk planes. (Nitrogen adsorbed on graphite is such an example. ${ }^{26}$ ) In this case the difference in the adsorption energies of adatoms at the surface and far from the surface is $-B$. The mismatch between the adatom configurations favored by the surface and the bulk causes a barrier in $V(h)$ whose height is parametrized by $A$. Thus it is more profitable to consider $A$ and $B$ as specifying the different kinds of interaction between substrate and interface and to consider separately the circumstances under which the various values of $A$ and $B$ arise.

In sum, we consider the initial Hamiltonian to be given by

$$
H=\sum_{\langle i j\rangle} H\left(h_{i}, h_{j}\right)
$$

with

$\boldsymbol{H}\left(h_{i}, h_{j}\right)=\left|h_{i}-h_{j}\right|+\left[V\left(h_{i}\right)+V\left(h_{j}\right)-\Delta \mu\left(h_{i}+h_{j}\right)\right] / 6$

and

$$
V(h)= \begin{cases}-A / h^{2}-B, & h>0, \\ 0, & h=0 .\end{cases}
$$

\section{RESULTS}

\section{A. Wet at zero temperature}

In this regime, the interface potential Eq. (2.13) is characterized by $A<0$ and $B>0$. The former ensures that $V(h)$ is a monotonically decreasing function of $h$ for $h>0$ which has the consequence that layer $n+1$ is energetically more favorable than layer $n$. This property is referred to as "sequencing" in Ref. 1. The latter condition has the consequence that the adsorption of an infinitely thick wetting layer is energetically favorable to no adsorption at all. The result of the two together is that the substrate is wet at zero temperature. In terms of the underly-

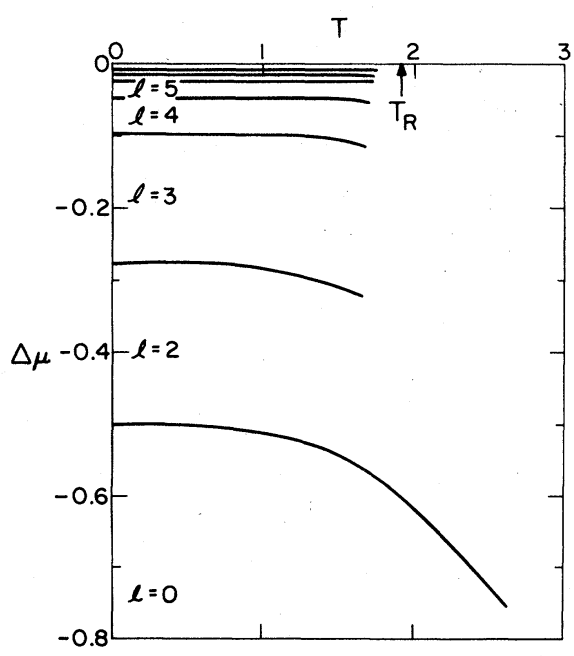

FIG. 1. Phase diagram of a system wet at zero temperature. Parameters in the potential of Eq. (2.13) are $A=-2, B=1.5$. The coverages at low temperatures are noted, as is the roughening temperature $T_{R}$. ing forces, these conditions have the interpretation that the substrate-adatom forces dominate the adatom-adatom forces. A first-principles calculations ${ }^{21}$ of $V(h)$ in terms of such forces can be used to define the conditions for such dominance.

Away from coexistence, the chemical potential must be taken into account. At zero temperature, the energy $\sum_{i}\left[V\left(h_{i}\right)-\Delta \mu h_{i}\right]$ can be minimized directly. One obtains an infinite sequence of first-order transitions between different layers as coexistence is approached, with the layer thickness $l \equiv\left\langle h_{i}\right\rangle$ diverging as $|\Delta \mu|^{-1 / 3}$. The RG equations display zero-temperature "sink" fixed points, one associated with each layer. Such sinks are characterized by the Boltzmann weights $x^{*}(h, k)=0$ except for the single diagonal entry $x^{*}(n, n)=1$, where $n$ is the integer closest to $l$. First-order transitions between layers $n$ and $m$ are characterized by zero-temperature discontinuity fixed points $x^{*}(h, k)=0$ except for the two diagonal entries $x^{*}(n, n)=x^{*}(m, m)=1$. At such points, there is one relevant field in the chemical potential direction which has associated with it an eigenvalue $y$ equal to the dimensionality of the surface, $y=2$.

Each of the infinite sequence of first-order layer transitions terminates at finite temperature at a critical point (see Fig. 1). The fixed point characterizing the critical point between layers $m$ and $n$ has the form $x^{*}(h, k)=0$ except for the $2 \times 2$ sub-block $x^{*}(n, n)=x^{*}(m, m)=1$, $x^{*}(n, m)=x^{*}(m, n) \simeq 0.54$. Note that there is an infinite number of such points but that they all have the identical form. Further, since only a $2 \times 2$ sub-block of $x^{*}(h, k)$ is nonzero, the transition is necessarily Ising-type.

As coexistence is approached, the sequence of layer critical temperatures approaches a limit temperature which we find to be $T_{1} \lesssim 1.9$ (see Table I). Plausible arguments ${ }^{12}$ identify this temperature with the roughening temperature $T_{R}$ of the bulk adsorbate, an identification which is supported by Monte-Carlo calculations ${ }^{13}$ and a mean-field scheme which incorporates roughening. ${ }^{17}$ To test this identification within our RG approach, we need to identify the roughening temperature. As is well known, the roughening transition is characterized by a fixed line which attracts RG flows for $T>T_{R}$ and repels them otherwise. ${ }^{27}$ There is a Kosterlitz-Thouless transition at $T_{R}$ itself. The Migdal approximation does not produce an exact fixed line, but an approximate one to which renormalization-group flows which originate at sufficiently high temperatures are attracted. The flows are essentially stationary for a large number of steps which de-

TABLE I. Critical temperatures and chemical potentials of the transition between layers $n$ and $n+1$.

\begin{tabular}{rrc}
\hline \hline$n$ & $T_{c}$ & $\Delta \mu$ \\
\hline 2 & 1.66 & -0.322 \\
3 & 1.68 & -0.113 \\
4 & 1.70 & -0.051 \\
5 & 1.72 & -0.027 \\
9 & 1.764 & $-4.9 \times 10^{-3}$ \\
14 & 1.790 & $-1.3 \times 10^{-3}$ \\
19 & 1.804 & $-5.5 \times 10^{-4}$ \\
39 & 1.832 & $-6.5 \times 10^{-5}$ \\
\hline \hline
\end{tabular}


TABLE II. As a function of the inverse temperature $K$, we display the values of $g^{(m)}$ and $m$, the smallest integer for which the relative error in the approximation $(2.10 \mathrm{~b})$ to the Gibbs potential (2.10a) is of order $10^{-16}$. The last column shows $\Delta=\left|g^{(m)}-g^{(m-1)}\right|$, a measure of the rate of change at $m$. Note that $g^{(m)}=1$ at the zero-temperature sink of the smooth phase; $g^{(m)}>1$ indicates roughening. The interface is assumed to be at infinity.

\begin{tabular}{lllc}
\hline \hline$K$ & $m$ & $g^{(m)}$ & $\Delta$ \\
\hline 1.0 & 6 & 1 & \\
0.9 & 7 & 1 & \\
0.8 & 7 & 1 & \\
0.7 & 8 & 1 & \\
0.6 & 11 & 1 & \\
0.55 & 16 & 1 & \\
0.54 & 19 & 1 & $4 \times 10^{-2}$ \\
0.53 & 23 & 1 & $1 \times 10^{-2}$ \\
0.52 & 26 & 1.07 & $3 \times 10^{-3}$ \\
0.51 & 24 & 1.40 & $6 \times 10^{-3}$ \\
0.50 & 23 & 1.52 & $3 \times 10^{-4}$ \\
0.49 & 22 & 1.60 & $5 \times 10^{-4}$ \\
0.48 & 22 & 1.66 & $5 \times 10^{-5}$ \\
0.47 & 21 & 1.72 & $5 \times 10^{-7}$ \\
0.46 & 21 & 1.78 & \\
0.45 & 20 & 1.84 & 2.15 \\
0.4 & 16 & & \\
\hline \hline
\end{tabular}

pends on the temperature, but eventually they go to a trivial sink. We define $T_{R}$ operationally within the Migdal approximation as follows. We calculate the free energy by following the renormalization of the constant part of the Hamiltonian. For $T>T_{R}$ the calculation of this free energy converges to within machine accuracy while the flows are still essentially stationary at the fixed line. This definition of $T_{R}$ is rather insensitive to the definition of "machine accuracy" and "essentially stationary." For details, see Table II. The data there show that the Migdal recursion relations indicate a roughening temperature at $T \simeq 1$.9. This confirms the identification of the limit of the layering critical points with $T_{R}$.

The way in which the layer critical temperatures approach the roughening temperature can be obtained from the following. Consider a discrete Gaussian model in the presence of a field which favors two heights $\hat{h}$ and $\hat{h}+1$ equally:

$$
H=\sum_{\langle i j\rangle}\left(h_{i}-h_{j}\right)^{2}+b \sum_{i}\left(h_{i}-\hat{h}-\frac{1}{2}\right)^{2} .
$$

For zero fields, the correlation length diverges as ${ }^{28}$

$$
\begin{aligned}
& \quad \xi \sim \exp \left(c t^{-1 / 2}\right), t=T_{R}-T, \\
& \text { while }^{29} \text { at } t=0, \\
& \xi \sim b^{-1 / 2},
\end{aligned}
$$

so that the critical curve has the form

$$
t \sim 1 /(-\ln b)^{2} .
$$

The potential of our case, $-A / h^{2}-B-\Delta \mu h$, can be set in the form of the above potential by expanding it about the minimum at $\Delta \mu(\hat{h})=2 A / \hat{h}^{3}$ to obtain $b=-6 A / \hat{h}^{4}$.

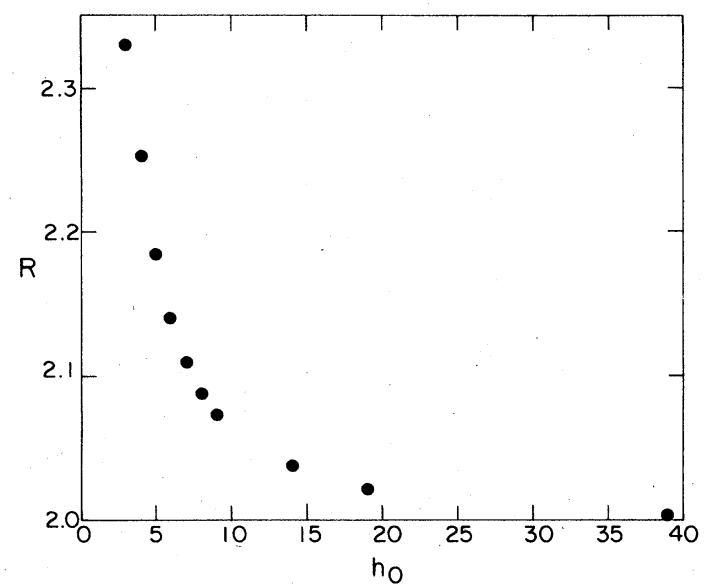

FIG. 2. Ratio $R=\Delta \mu\left(h_{0}\right) /\left[\left(h_{0}+1\right)^{-2}-h_{0}^{-2}\right]$ evaluated at the critical temperature of the transition between layers $h_{0}$ and $h_{0}+1$ is plotted versus $h_{0}$. The parameters in the potential are $A=-2, B=1.5$. The value of $R$ determined by energy considerations above is $-A=2$.

Thus the difference $t(h)$ between the layer critical temperature and the roughening temperature should behave as

$$
t(h) \sim 1 /\{-\ln [\Delta \mu(h)]\}^{2} .
$$

This indicates that the roughening temperature is somewhat above a naive linear extrapolation of the critical temperatures. Also,

$$
\xi \sim(|\Delta \mu|)^{-2 / 3}
$$

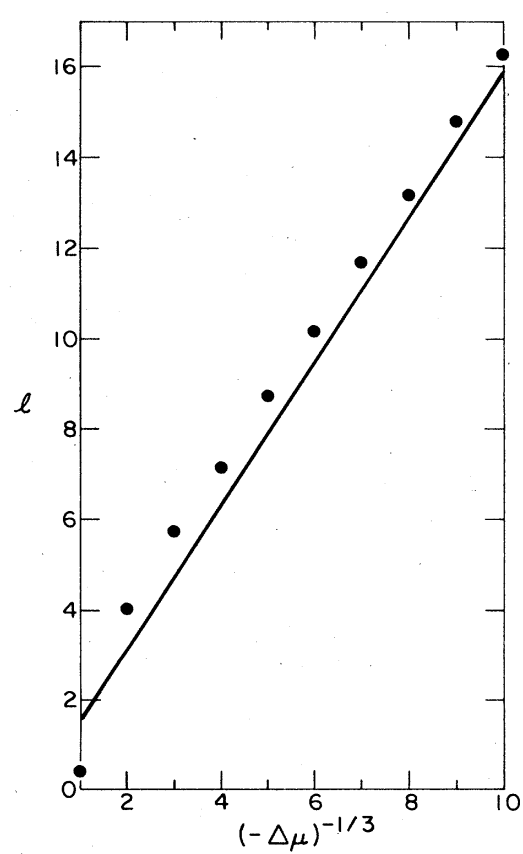

FIG. 3. The way in which the film thickness $l$ diverges as coexistence is approached at $T=3$ which, for this potential $A=-2, B=1.5$, is above the wetting temperature, $T=0$, and above the roughening temperature, $T_{R}=1.9$. Calculated points are shown. The solid line is the result expected from energy considerations alone, $l=(2 A \Delta \mu)^{1 / 3}$. 
is an immediate consequence of the above. ${ }^{30}$

In deriving Eq. (3.3), we assumed that the chemical potential along the $(\hat{h}, \hat{h}+1)$ first-order layer transition line does not change with temperature and therefore is given by its zero-temperature value. Our calculations show that fluctuations only slightly modify this behavior quantitatively: Asymptotically the exponent, and even the amplitude, remains unchanged. This is illustrated in Fig. 2 where the quantity $\Delta \mu(\hat{h}) /\left[(\hat{h}+1)^{-2}-\hat{h}^{-2}\right]$ is plotted versus $\hat{h}$ at the layer critical temperatures for $A=-2$ and $B=1.5$. Note that at $T=0$ this ratio equals $-A$. Figure 3 shows that this zero-temperature behavior also obtains at temperatures even well above $T_{R}$. There the relation between film thickness and chemical potential is seen to be the same as obtained from energy considerations alone; i.e., $l \sim(-\Delta \mu)^{-1 / 3}$. This is in agreement with mean-field theory. ${ }^{11}$ Deviations occur only for very thin films.

Equation (3.3) predicts the layer critical temperatures to approach the roughening temperature from below. This is physically plausible and can be seen as follows. Include in the Hamiltonian (3.1) a term $\Delta \sum_{i} h_{i}$, and define

$$
h(b, \Delta)=\left\langle h_{i}-\hat{h}-\frac{1}{2}\right\rangle .
$$

It is reasonable to assume that

$$
\left.\left|h\left(b_{1}, \Delta\right)\right|\right\rangle\left|h\left(b_{2}, \Delta\right)\right|
$$

for $b_{1}<b_{2}$, reflecting the fact that the restriction on the column heights becomes less stringent as $b$ decreases and that the magnitude of $h(b, \Delta)$ diverges as $b$ decreases to 0 . Since the inequality (3.4) then also holds for the order parameter, the shifted film height in the limit of vanishing $\Delta$, it follows that the critical temperature increases as $b$ decreases, i.e., as the layer number goes up. This prediction agrees with the results of Ref. 17 and can be tested in those systems in which many-layer transitions have been reported. $^{31}$ It agrees with experimental results of solid ${ }^{4} \mathrm{He}$ on graphite. ${ }^{31}$

We next consider the question of whether all layers are encountered as coexistence is approached or whether some are missing. Such a case is shown in Fig. 1 where layer 1 is missing. It is easy to show that the particular sequence of layers encountered depends upon the particular interface potential. For the one we have chosen, Eq. (2.13), simple energy arguments show that the only possible sequence is $(0, n+1, n+2, \ldots)$ so that layers 1 to $n$ are missing. This sequence occurs at zero temperature when $|A| / n^{2}>B>|A| /(n+1)^{2}$. (All layers occur for $B>|A|)$. The appearance of the phase diagram depends strongly on the magnitudes of $B$ and $A$ relative to the energy needed to create a step of unit height between successive layers, an energy which we have arbitrarily set to unity. Figure 4 shows results for the specific case in which the initial layer transition at zero temperature is between layers 0 and 2 .

When $|A|$ and $B$ are small compared to the step energy [Figs. 4(a) and 4(d)], layer 1 is not seen at low temperatures but appears at high temperatures. This is because the entropy makes the energetically unfavorable layer 1 become free-energetically favorable for temperatures of the order of $V(1)-V(0) \simeq V(1)-V(2)$. There is a triple point where the three phases, layer heights of 0,1 , and 2 meet. This point flows to a zero-temperature fixed point $x(h, k)^{*}$ which vanishes everywhere except for the three unit diagonal elements corresponding to the three different layers. There are two relevant scaling fields each with eigenvalue $y=2$. As the magnitudes of $A$ and $B$ are increased, the phase boundary between layers 0 and 1 becomes smaller and eventually disappears at a critical end point. [A separatrix between RG's flows to the zero-layer sink and one-layer sink is then observed as shown in Fig. 4(b). No singularities in the free energy are encountered on crossing such a separatrix, of course.] The shape of the density-temperature phase boundary reflects the incipient 0-1 critical point. ${ }^{32}$ For $|A|, B$ larger than the step energy, the unfavorable layer 1 is statistically improbable and the system behaves as if the basic step were between layers 0 and 2, a double step which costs twice the energy of a single step. As this double-step energy sets the scale of the 0-2 critical point, its temperature is roughly twice that of the transitions between successive layers. This latter case is shown in Figs. 4(c) and (4e) and also in Fig. 1. The curvatures apparent in Fig. 1 are related to the en-

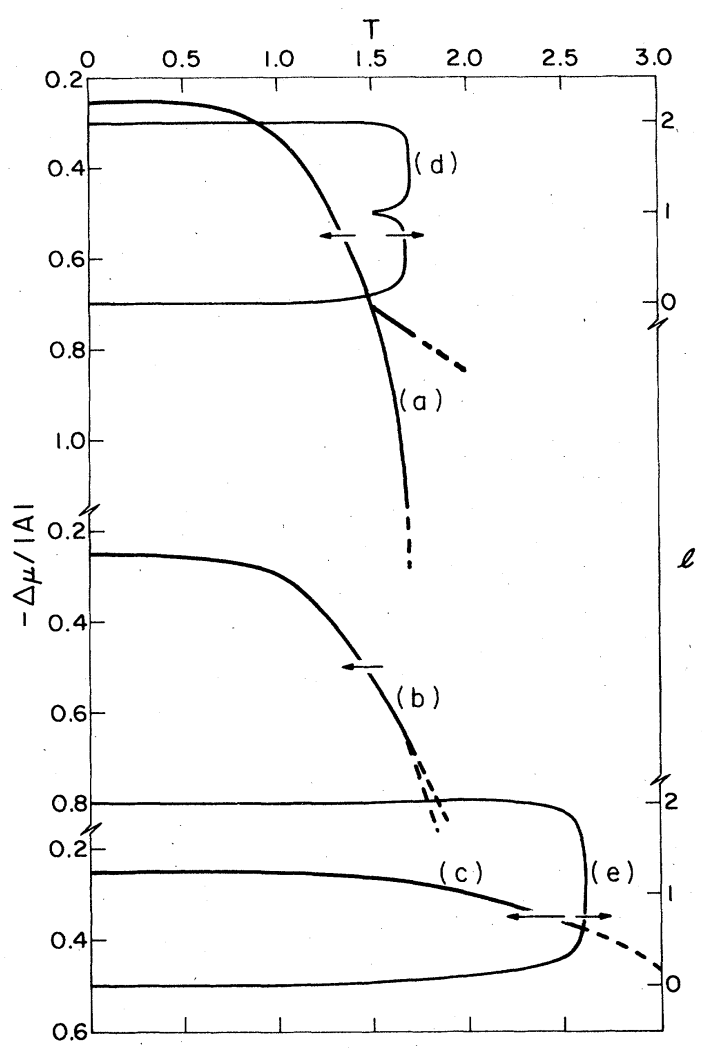

FIG. 4. (a) Transition between layers 0 and 2 is shown in solid line in the $\Delta \mu-T$ plane. Layer 1 appears above $T=1.5$ and there is a triple point. Dotted lines show separatrices in RG flow. Parameters in the potential are $A=-0.1, B=0.075$. (b) Parameters of the potential are $A=-0.175, B=0.131$. Layer 1 no longer appears, but separatrices between RG flows to sinks of layers 0,1 , and 2 are observed and are shown by dotted lines. (c) Parameters of the potential are $A=-2, B=1.5$, as in Fig. 1. (d) Same transition as in (a) is shown in $l-T$ plane. (e) Same transition as in (c) is shown in $l-T$ plane. 
tropies per unit area of each phase, $s$, and their thicknesses $l$ according to 8

$$
\left.\frac{d \mu}{d t}\right|_{\text {boundary }}=-\frac{s_{m}-s_{n}}{l_{m}-l_{n}} .
$$

The reasonable assumption that thicker films have more entropy than thinner ones leads to the negative slopes observed in the figure.

As the value of $B$ is made smaller, more and more layers disappear from the zero-temperature sequence. Finally as $B$ changes sign, there is no longer an infinitely thick wetting layer adsorbed at zero temperature.

\section{B. Not wet at zero temperature}

With the parameters $A$ and $B$ both negative, the interface potential, Eq. (2.13), exhibits a potential barrier between no adsorption, $V(0)=0$, and adsorption of one layer, $V(1)=|A+B|$. Beyond $h=1, V(h)$ is monotonically decreasing to $V(\infty)=|B|$. Thus the lowest energy state is one in which $h$ is finite (zero for our particular choice of potential) so that the substrate is not wet at zero temperature. However, once the barrier to adsorption of one layer has been overcome entropically, adsorption of an infinitely thick wetting layer is more probable than that of a finite layer. Thus a first-order-wetting transition takes place.

The RG calculation in this regime involves only one new fixed point, that which describes the first-order wetting. Due to the first-order nature of the transition, the fixed point is an ordinary discontinuity fixed point between two layers, one with a finite layer number and the other with what should be an infinite layer number. In our approximate calculation in which column heights cannot exceed some cutoff value $h_{\max }$, the "wetting layer" number increases linearly with this cutoff. Its precise value depends upon the conditions imposed at the artificial boundary at $h_{\max }$. The phase diagram encountered depends upon whether the wetting temperature $T_{W}$ is lower than $T_{R}$ (layering subregime) or higher than $T_{R}$ (prewetting subregime).

\section{Layering subregime and the low-temperature series}

A typical example of a phase diagram in this subregime is shown in Fig. 5 for $A=-1$ and $B=-0.1$. Transitions between low layer numbers have been replaced by a single first-order transition ending in a critical point which is Ising-type. This is completely analogous to what is seen in Fig. 4, where the two transitions 0-1 and 1-2 are replaced by a single transition. Near coexistence, firstorder transitions occur between higher layers which have sufficiently small energy differences so that entropy considerations make such transitions free-energetically favored. At high temperatures, these transitions end in critical points with $T_{R}$ as a limit; at low temperature they end in triple points with $T_{W}$ as a limit. The location of the first-order wetting temperature can be obtained from a low-temperature series expansion. This expansion is most easily generated by considering the Gibbs potential $\Omega(\mu, T)$ defined by

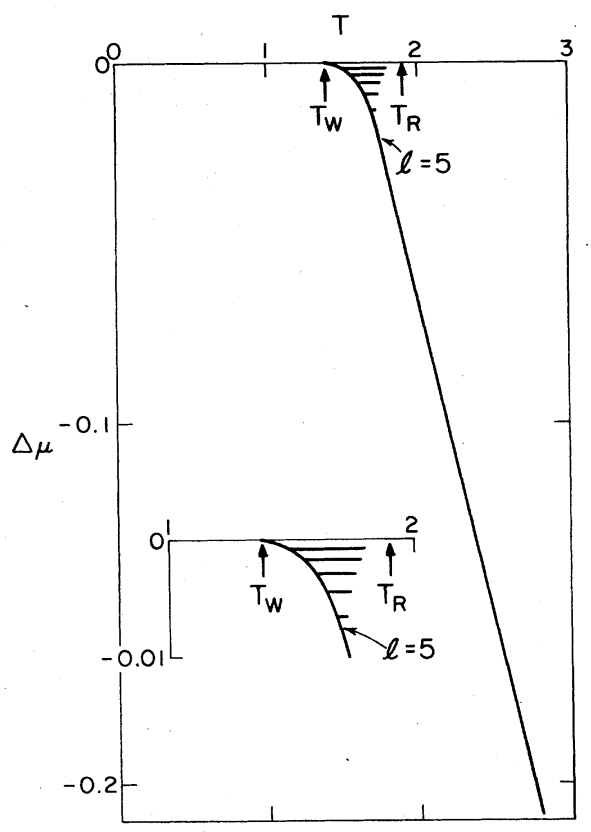

FIG. 5. Phase diagram for a system with a first-order wetting transition at a temperature below the roughening temperature. Potential parameters are $A=-1, B=-0.1$.

$\exp [N \Omega(\Delta \mu, T)] / T=\sum_{M=0}^{\infty} \exp \{-[F(M, T)-\Delta \mu M] / T\}$,

where $N$ is the number of lattice sites of the SOS model, and $F(M, T)$ is the Helmholtz free energy of a system with fixed total column height

$$
M=\sum_{i} h_{i} \text {. }
$$

We assume that the sum is dominated by those terms in which the total column height is either equal to $M_{0}=N n$, with $n$ integer, or equal to $M_{0} \pm 1$. Further, the free energy $F(M, T)$ is approximated by the energy $F(M, 0)$. With these assumptions,

$$
\begin{aligned}
\Omega_{n}(\Delta \mu, T) \simeq & -V(n)+n \Delta \mu \\
& +T \exp \{-[V(n+1)-V(n)+6-\Delta \mu] / T\} \\
& +T \exp \{-[V(n-1)-V(n)+6+\Delta \mu] / T\}
\end{aligned}
$$

where the factor of 6 is the number of nearest neighbors of the triangle lattice. Using the explicit form of the potential $V$ and assuming $1 \ll n$ and $\Delta \mu \sim A / n^{3} \ll 1$, we simplify this to

$$
\Omega_{n}(\Delta \mu, T) \simeq A / n^{-2}+B+n \Delta \mu+2 T e^{-6 / T} .
$$

If $n=0$, however, which refers to the bare substrate, then only the term $M=0$, and the value 1 are included in the sum so that

$$
\Omega_{0}(\Delta \mu, T) \simeq T e^{-[6-(A+B)] / T} .
$$

At a first-order transition between the bare substrate and a film $n$ layers thick, the Gibbs potentials $\Omega_{n}(\Delta \mu, T)$ and $\Omega_{0}(\Delta \mu, T)$ are equal while their first derivatives are not. 
The equality of the potentials yields the transition temperature $T_{n}(\Delta \mu)$

$$
T_{n}(\Delta \mu) \simeq T_{w}\left[1+\frac{T_{w}}{6 n^{2}}\left|\frac{A}{B}\right|-\frac{T_{w} n \Delta \mu}{6|B|}\right),
$$

where $T_{w}$ is the first-order wetting temperature which occurs for $\Delta \mu=0$ and $n \rightarrow \infty$,

$T_{w} \simeq 6 /\left(\ln (12 /|B|)+\ln \left\{1-\frac{1}{2} \exp [(A+B) / 6]\right\}\right)$.

We note that this expression depends only weakly on the parameters of the potential.

It is interesting to observe that the difference in Gibbs potentials $-\left[\Omega_{n}(\Delta \mu, T)-\Omega_{0}(\Delta \mu, T)\right]$ can be written in precisely the same form as the energy difference $V(n)-V(0)-n \Delta \mu=-A / n^{2}-B-n \Delta \mu$, but with a temperature-dependent $B$; that is,

$$
-\left[\Omega_{n}(\Delta \mu, T)-\Omega_{0}(\Delta \mu, T)\right]=-A / n^{2}-\widetilde{B}(T)-n \Delta \mu,
$$

where $\widetilde{B}(T)=B+2 T e^{-6 / T}\left[1-\frac{1}{2} e^{(A+B) / T}\right]$. Just as $|B|$ is the difference between the energies of the absolute and local minima at zero and infinite coverages, so $\widetilde{B}$ is the difference between the local maxima of the Gibbs potentials. A first-order transition occurs when this difference vanishes.

From Eq. (3.6) for $T_{n}(\Delta \mu)$ we can immediately determine that the slope of the line of first-order transitions between 0 and $n$ layers is given by $d(\Delta \mu) / d t=6|B| / T_{w} n$ and vanishes as coexistence is approached and $n$ diverges. This is in accord with the general thermodynamic relation Eq. (3.5). Moreover, since $\Delta \mu \simeq 2 A / n^{3}$ at low temperatures, this equation can be integrated to yield

$$
T-T_{w}=T_{w}^{2}|A|^{1 / 3} \Delta \mu^{2 / 3} / 2|B| \text {. }
$$

As can be seen from Eq. (3.7), the first-order-wetting temperature increases as the parameter $B$ becomes more negative. Eventually, one can make $T_{w}$ of the order of, or exceed, the roughening temperature $T_{R}$. In this case there is no longer an infinite series of layering transitions, but only a simple thin-film-thick-film line of first-order transitions. This defines the prewetting subregime.

\section{Prewetting subregime}

The line of first-order transitions between thin and thick films is called the prewetting line. Away from coexistence, this line ends in an ordinary two-dimensional Ising critical point. It approaches the coexistence line as

$$
T-T_{w} \sim|\Delta \mu|^{2 / 3}
$$

just as in the layering subregime [cf. Eq. (3.8)]. Again this result follows from thermodynamics and the form of the van der Waals potential. The first-order-wetting temperature is again given rather well by Eq. (3.7). Figure 6 shows an example of a phase diagram in this regime for the case $A=-3$ and $B=-0.3$. The same results plotted as $|\Delta \mu|^{2 / 3}$ versus $\left(T-T_{w}\right)$ is also shown in the figure. The parameter $A$ hardly affects the location of $T_{w}$ as can be seen from Eq. (3.7), but it does affect the location of the prewetting critical point. As $A$ is made less negative,

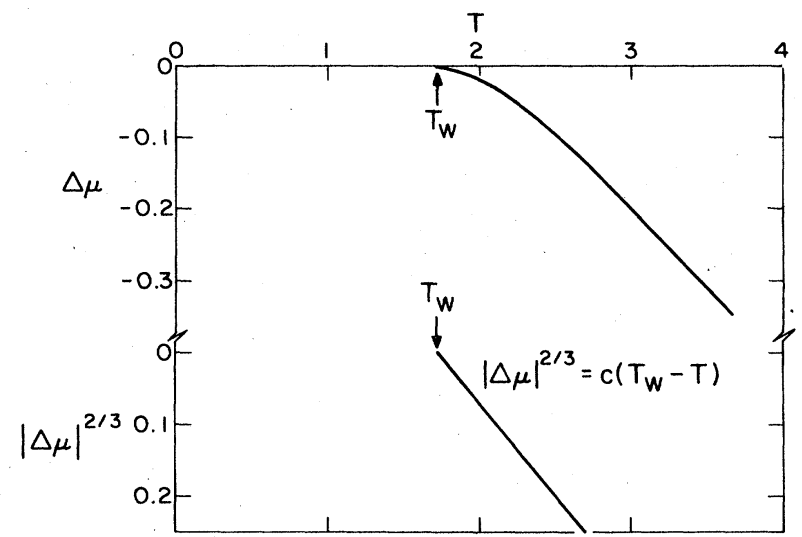

FIG. 6. Phase diagram for a system in prewetting subregime is shown with $T$ as a function of $\Delta \mu$ and $|\Delta \mu|^{2 / 3}$. Near coexistence, it is a straight line in the latter, with slope $c$, a constant. Potential parameters are $A=-3, B=-0.3$.

the length of the prewetting line shrinks. This is clearly seen by comparing Figs. 6 and 7. In the latter, $A$ has been reduced by a factor of 3 and $B$ has been adjusted so that $T_{w}$ is unchanged.

The next region of interesting behavior occurs when $A$ actually changes sign, so that $V(h)$ is nonsequencing. This is the region in which critical wetting is expected to occur if it occurs at all.

\section{Critical wetting subregime}

The potential $V(h)$ in this regime is characterized by a monotonic increase with $h$ for large $h$. With our choice, Eq. (2.13), $A$ is positive. This arises from a physical situation in which thicker films are always more expensive energetically than thinner ones. The film thickness will increase with temperature for entropic reasons. The crucial question is whether the increased entropy outweighs the increased energy. If so, critical wetting can occur; if not, critical wetting cannot occur. It has been shown via RG arguments that critical wetting can occur if the forces are short ranged, ${ }^{4,5}$ and we have verified this using our SOS model. ${ }^{2}$ However, for the experimentally interesting case of long-range forces, we have found that critical wetting is not possible. ${ }^{2}$ All of our results for interactions of whatever form are consistent with a Helmholtz potential per site of the form

$F(T, h) / N=V(h)-T\{S(\infty)-C \exp [-h / \xi(T)]\}+\cdots$,

where terms which vanish more rapidly with $h$ have been

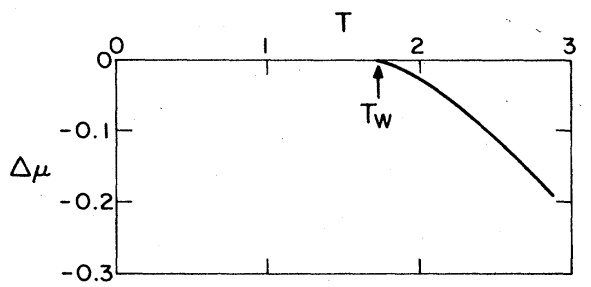

FIG. 7. Phase diagram for a system in the prewetting subregime. Potential parameters are $A=-1, B=-0.24$. 
ignored. In this expression $S(\infty)$ is a constant, $C$ can be temperature dependent, and $\xi(T)$ a length which is a linear function of temperature. Minimization of $F(T, h)$ yields the equilibrium film thickness $l(T)$. In particular, we find no critical wetting for long-range potentials of the form

$$
V(h)= \begin{cases}-|A| / h^{\sigma}-B, & h>0, \\ 0, & h=0 .\end{cases}
$$

As noted earlier, van der Waals forces correspond to $\sigma=2$. Strain in a solid film gives rise to a potential of the above form ${ }^{21,22}$ with $\sigma=1$. The results of this section can also be obtained from a low-temperature series expansion as shown in the Appendix.

Although there is no wetting transition with potentials of the form of Eq. (3.10), there can be a first-order transition from a thin film to a finitely thick film at coexistence. A line of such transitions extends into the $\Delta \mu-T$ plane much like a prewetting line. The difference is that, in this case, the thick film remains of finite thickness even at coexistence. A thermodynamic consequence of this is that the slope of the first-order line approaches coexistence with a finite slope, which can be obtained from Eq. (3.5).

Whether there is or is not such a first-order jump in the film thickness depends on the parameters $A$ and $B$. We find that for $B$ positive, there is such a transition for sufficiently large positive $A$, whereas if $B$ is negative, there is such a transition for all $A$.

Lastly, we briefly discuss the applicability of our results obtained in an SOS model to lattice-gas models or experimental systems. One feature of the latter which is not contained in the SOS model is the variation in the density of the adsorbed and bulk phases with temperature, a variation which becomes quite large as a liquid-gas critical point is approached. As noted earlier, the SOS model does not contain such a point. However, the manner in which the model can be modified to include these entropic effects is already indicated in Sec. III B1. There we noted that the difference in Gibbs potentials $-\left[\Omega_{n}(\Delta \mu, T)\right.$ $\left.-\Omega_{0}(\Delta \mu, T)\right]$ had the same form as the zero-temperature energy difference $V(n)-V(0)-n \Delta \mu$ but with a temperature-dependent $B$. This temperature dependence arises from the entropic effects of the interface, which are described by the SOS model. This suggests that those entropic effects which are not explicitly included in the SOS model can be implicitly included by replacing the potential $V(h)$ by an effective one with temperature-dependent constants $A$ and $B .^{33}$ Furthermore, from our remarks above, it follows that the equilibrium properties can be obtained from a minimization of Eq. (3.9) with the effective potential replacing $V(h)$. Such entropic effects, which do not arise from the interface or its fluctuations can also bring about a wetting transition. ${ }^{33}$ For example, assume the long-range form of the effective potential is of the form

$$
V_{e}(h, T)=-A_{e}(T) / h^{\sigma}-C_{e}(T) / h^{\tau}-\left|B_{e}(T)\right|
$$

with $\tau>\sigma$ and $A_{e}$ positive, as in the discussion above, only for $T$ less than $T_{0}$ and negative for $T$ greater than $T_{0} . C_{e}$ is assumed to be of one sign. If $C_{e}$ were positive, a first-order transition could take place above $T_{0}$. If $C_{e}$ were negative a continuous transition would take place at $T_{0}$ at coexistence. Writing $A_{e}(T)=-|a| t$, with $t \equiv T-T_{0}$, we see that $t$ plays the same role in this transition as the chemical potential difference plays as coexistence is approached above $T_{W} \cdot{ }^{11}$ The critical properties can be obtained in the same way as in that case treated in Sec. III A. We find that the field $b$ of Eq. (3.1) behaves like $b \approx|t|^{(\tau+2) /(\tau-\sigma)}$ so that

$$
\begin{aligned}
\xi & \sim b^{-1 / 2} \\
& \approx|t|^{-v}, \quad v=(\tau+2) / 2(\tau-\sigma) .
\end{aligned}
$$

The behavior of the film thickness follows from a minimization of (3.9)

$$
l \approx|t|^{-1 /(\tau-\sigma)}
$$

from which one obtains the behavior of the free energy, also from (3.9)

$$
f \approx|t|^{2-\alpha}, \quad \alpha=(\tau-2 \sigma) /(\tau-\sigma) .
$$

These results are completely analogous to those obtained in Ref. 11.

\section{SUMMARY}

The global nature of the phase diagram of an adsorbed system and the particular question of whether that phase diagram contains a wetting transition both depend upon the interface potential $V(h)$, but in different ways. The existence and nature of the wetting transition depend solely on the asymptotic behavior of $V(h)$. From what is already known about this potential, and from the work above, the following general statements can be made.

(1) There can be no wetting transition along the gassolid or liquid-solid phase boundaries as coexistence is approached from the low-density side. This follows from the fact that $V(h)$, in the case of solid films, is attractive and falls off ${ }^{21,22}$ like $1 / h$ and that for such potentials there can be no wetting (Sec. III B3). There can exist transitions between thin and very thick films, of course, which would be experimentally difficult to distinguish from wetting. We believe that the transition on the solid-gas phase boundary which has recently been reported ${ }^{34}$ as a wetting transition in the $\mathrm{CF}_{4} /$ graphite system is, in fact, of this type.

(2) The mechanism we have studied, in which wetting is brought about by interface fluctuations can only produce a first-order wetting transition along the gas-liquid coexistence line; it cannot produce a continuous wetting. This follows from the fact that, in the case of liquid films, $V(h)$ falls off like $1 / h^{2}$. Whether the potential is attractive or repulsive depends on the relative strengths of the adatom-adatom and adatom-substrate potentials. For strong substrates, $V(h)$ will be repulsive, and it should be possible to observe a first-order wetting. For weak substrate potentials, $V(h)$ should be attractive so that interface fluctuations cannot produce wetting. However, simple entropic effects which are unrelated to interface fluctuations, such as the temperature dependence of the liquid density, can produce wetting along the gas-liquid coexistence. 
(3) There can be a continuous wetting at the triple point. ${ }^{14,35}$ This follows from the fact that, for very strong substrates, we expect that the wetting transition on the gas-liquid coexistence, which would take place at a very low temperature, will be preempted by the formation of solid. In this case the substrate is wet by the liquid as the gas-liquid phase boundary is approached at any temperature between the triple temperature $T_{t}$ and the gas-liquid critical point, so that there is no wetting transition in this temperature range. However, since the substrate cannot be wet by the solid at any temperature below $T_{t}$, the triple temperature itself must play the role of the wetting temperature. $^{36,37}$ At this temperature, the wetting transition is continuous but is not critical wetting; the behavior of the film as $T_{t}$ is approached is precisely the same as in the approach to any other point on the gas-liquid coexistence curve. In particular the film thickness diverges as $|\Delta \mu|^{-1 / 3}$. As $|\Delta \mu|$ and $T_{t}-T$ are linearly related along a smooth path approaching $T_{t}$, one also finds that the thickness diverges as $\left(T_{t}-T\right)^{-1 / 3}$ This triple-point wetting appears to be common. ${ }^{37}$

The global phase diagram depends on $V(h)$ for all $h$, not just its asymptotic value. If $V(h)$ is initially a decreasing function of $h$, as in Sec. III A, we expect a series of layer transitions. The long-range attractive part of $V(h)$ due to strain will have the effect of making the number of layer transitions finite instead of infinite. If the strain is small, the layer transitions will be cut off at a very large layer number. As transitions between such thick films are difficult to observe experimentally, there would be little effect observationally. Similarly, in cases in which $V(h)$ has an initial barrier, we expect a firstorder thin-thick film transition as in Sec. III B 1. The effect of a strain would be to make the thick film finite at coexistence, and the line of first-order transitions approach coexistence with a finite slope. The general form of the phase diagram would be qualitatively similar to that of Fig. 5 or 6.

In conclusion, from a knowledge of the interface potential $V(h)$, which can be calculated from first principles in some simple cases, much can be said with certainty concerning a possible wetting transition, and much can be anticipated about the global phase diagram.

\section{ACKNOWLEDGEMENTS}

We thank A. N. Berker, J. G. Dash, S. Dietrich, C. Ebner, and M. Wortis for stimulating and informative conversations. This work was supported in part by the National Science Foundation under Grant Nos. DMR79-20785, DMR-83-19301, and DMR-84-06186.

\section{APPENDIX}

We use a low-temperature series expansion to determine the temperature at which a transition from $n$ to $n+1$ layers is made at coexistence, $\Delta \mu=0$. An expansion of the Gibbs potential assuming layer $n$ to give the dominant contribution can be written

$$
\exp \left[N \Omega_{n}(0, T) / T\right]=\exp [-N V(n) / T] Z(n),
$$

where $Z(n)$ is the partition function in which energies are measured with respect to $V(n)$. Similarly, an expansion about layer $n+1$ can be written

$\exp \left[N \Omega_{n+1}(0, T) / T\right]=\exp [-N V(n+1) / T] Z(n+1)$.

There are two kinds of terms in $Z(n+1)$. First are those, representing fluctuations about an interface at layer $n+1$, which have counterparts in $Z(n)$ representing the same configuration of fluctuations about an interface at layer $n$. For temperatures small compared with the step energy and for potentials such that $V / T$ is also small, these terms can be ignored for algebraic potentials. Second are the terms representing fluctuations about an interface at layer $n+1$ which have no counterpart in $Z(n)$. These are terms involving excursions of the interface all the way to the substrate. The simplest such fluctuation has a Boltzmann weight $\exp [V(n+1)-6(n+1)] / T$, where 6 is the number of nearest neighbors of the triangular lattice. If we keep such terms, the difference in Gibbs potentials can be written

$$
\begin{aligned}
-\left[\Omega_{n+1}(0, T)-\Omega_{n}(0, T)\right]= & V(n+1)-V(n) \\
& -T e^{[V(n+1)-6(n+1)] / T}+\cdots
\end{aligned}
$$

which has the form

$$
-\delta \Omega(n)=\delta E(n)-T \delta S(n),
$$

where $\delta S$ depends exponentially on $n$. The transition temperature $T(n)$ is determined by setting $\delta \Omega=0$. For large $n,[V(n+1)-6(n+1)] \rightarrow-6 n$ as $V(\infty)$ is finite. Thus $T(n)$ is a solution of

$$
T(n)=\frac{6 n}{\left[-\ln \left[\frac{1}{T(n)} \frac{d V}{d n}\right]\right]} .
$$

For a long-range potential, $T(n)$ increases as $n / \ln (n)$, suggesting that there is no finite critical wetting temperature, $T(\infty)$. For short-ranged potentials, the analysis is considerably more involved, and we simply state the result that finite wetting temperatures are obtained.
${ }^{*}$ Present address.

${ }^{1}$ See R. Pandit, M. Schick, and M. Wortis, Phys. Rev. B 26, 5112 (1982), for a guide to the literature through most of 1982. More recent work is cited in Ref. 2.

${ }^{2}$ M. P. Nightingale, W. F. Saam, and M. Schick, Phys. Rev. Lett. 51, 1275 (1983).

${ }^{3}$ J. W. Schmidt and M. R. Moldover, in Proceedings of the Conference on Fronts, Interfaces, and Patterns, Los Alamos
National Laboratory, 1983 (unpublished).

${ }^{4}$ E. Brézin, B. I. Halperin, and S. Leibler, Phys. Rev. Lett. 50, 1387 (1983).

${ }^{5}$ R. Lipowsky, D. M. Kroll, and R. K. P. Zia, Phys. Rev. B 27, 4499 (1983).

${ }^{6}$ E. Brézin, B. I. Halperin, and S. Leibler, J. Phys. (Paris) 44, 775 (1983).

${ }^{7}$ P. Tarazona and R. Evans, Mol. Phys. 48, 799 (1983). 
${ }^{8}$ E. H. Hauge and M. Schick, Phys. Rev. B 27, 4288 (1983).

${ }^{9}$ R. Pandit and M. Wortis, Phys. Rev. B 25, 3226 (1982).

${ }^{10}$ G. F. Teletzke, L. E. Scriven, and H. T. Davis, J. Chem. Phys. 77, 5794 (1982) and 78, 1431 (1983).

${ }^{11}$ R. Lipowsky, Phys. Rev. Lett. 52, 1429 (1984).

${ }^{12}$ M. J. de Oliveira and R. B. Griffiths, Surf. Sci. 71, 687 (1978).

${ }^{13}$ I. M. Kim and D. R. Landau, Surf. Sci. 110, 415 (1981).

${ }^{14}$ C. Ebner, Phys. Rev. B 28, 2890 (1983).

${ }^{15}$ C. Ebner, C. Rottman, and M. Wortis, Phys. Rev. B 28, 4186 (1983).

16W. F. Saam, Surf. Sci. 125, 253 (1983).

17J. D. Weeks, Phys. Rev. B 26, 3998 (1982).

18J. M. Luck, S. Leibler, and B. Derrida, J. Phys. (Paris) 44, 1135 (1983).

19J. D. Weeks, in Ordering in Strongly Fluctuating Condensed Matter Systems, edited by T. Riste (Plenum, New York, 1980).

20J. L. Seguin, J. Suzanne, M. Bienfait, J. G. Dash, and J. M. Venables, Phys. Rev. Lett. 51, 122 (1983).

${ }^{21}$ F. T. Gittes and M. Schick, Phys. Rev. B 30, 209 (1984).

22D. A. Huse, Phys. Rev. B 29, 6985 (1984).

${ }^{23}$ M. E. Fisher, S. K. Ma, and B. G. Nickel, Phys. Rev. Lett. 29, 917 (1972).

${ }^{24} \mathrm{~T}$. W. Burkhardt, in Real Space Renormalizaton, edited by T. Burkhardt and J. M. J. van Leeuwen (Springer, Berlin, 1982).

${ }^{25}$ We set Boltzmann's constant to unity.
${ }^{26}$ See R. D. Diehl and S. C. Fain, Jr., Surf. Sci. 125, 116 (1983).

${ }^{27}$ J. V. José, L. P. Kadanoff, S. Kirkpatrick, and D. R. Nelson, Phys. Rev. B 16, 1217 (1977).

28J. M. Kosterlitz, J. Phys. C 7, 1046 (1974).

${ }^{29} \mathrm{P}$. Pfeuty and G. Toulouse, Introduction to the Renormalization Group and to Critical Phenomena (Wiley, New York, 1975).

${ }^{30}$ These results have been obtained independently by $D$. A. Huse, Phys. Rev. B 30, 1371 (1984).

${ }^{31}$ See, for example, J. J. Hamilton and D. L. Goodstein, Phys. Rev. B 28, 3838 (1983), for a study of methane adsorbed on graphite; and S. Ramesh, Q. Zhang, G. Torzu, and J. D. Maynard, Phys. Rev. Lett. 52, 2375 (1984), for a study of solid helium adsorbed on graphite:

${ }^{32}$ D. M. Butler, J. A. Litzinger, G. A. Stewart, and R. B. Griffiths, Phys. Rev. Lett. 42, 1239 (1979).

${ }^{33}$ R. Lipowsky and D. M. Kroll, Phys. Rev. Lett. 52, 2303 (1984).

34J. Suzanne, J. L. Seguin, M. Bienfait, and E. Lerner, Phys. Rev. Lett. 52, 637 (1984).

${ }^{35}$ R. Pandit and M. E. Fisher, Phys. Rev. Lett. 51, 1772 (1983).

36J. Menaucourt, A. Thomy, and X. Duval, J. Phys. (Paris) Colloq. 38, C4-195 (1977).

37J. Krim, J. G. Dash, and J. Suzanne, Phys. Rev. Lett. 52, 640 (1984). 